\title{
Failed Colonoscopy due to Hernia
}

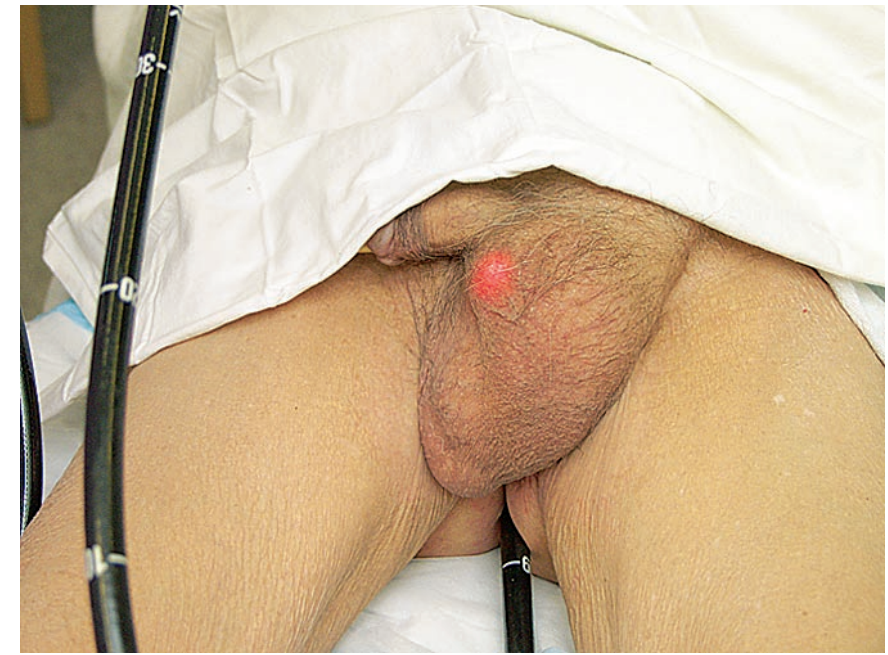

Figure 1 A 70-year-old man was referred for colonoscopy for the investigation of bleeding per rectum. No history of hernia was documented in the referral notes. Colonoscopy by the first endoscopist failed due to "acute looping in the sigmoid colon." The patient was examined by the second endoscopist, and after removal of the pubic covering it was found that the colonoscope was actually inside a hernia (light seen inside the left scrotum). The hernia was reduced and full colonoscopy was completed with manual compression being applied to the orifice of the hernia.

\section{Y. T. Lee, A. Y. Hui}

Department of Medicine and Therapeutics, Prince of Wales Hospital, Chinese University of Hong Kong, Shatin, Hong Kong, China

Corresponding Author

Y. T. Lee, M.D.

Department of Medicine and Therapeutics

The Chinese University of Hong Kong Prince of Wales Hospital Shatin, N.T.

Hong Kong SAR

China

Fax: + 852-2637-5396

E-mail: leeytong@cuhk.edu.hk 